\title{
An efficient Helmholtz solver for acoustic transversely
}

3

4

\section{isotropic media}

\author{
Zedong $\mathbf{W u}[1]$ and Tariq Alkhalifah[1] \\ [1] King Abdullah University of Science and Technology (KAUST), \\ Physical Science and Engineering Division (PSE), \\ Thuwal, 23955-6900, Saudi Arabia \\ E-mail: zedong.wu@kaust.edu.sa; tariq.alkhalifah@kaust.edu.sa
}

(November 9, 2017)

Running head: Efficient Helmoltz solver

\begin{abstract}
The acoustic approximation, even for anisotropic media, is widely used in current industry imaging and inversion algorithms mainly because P-waves constitute the majority of the energy recorded in seismic exploration. The resulting acoustic formulas tend to be simpler, resulting in more efficient implementations, and depend on less medium parameters. However, conventional solutions of the acoustic wave equation with higher-order derivatives suffer from S-wave artifacts. Thus, we propose to separate the quasi-P wave propagation in anisotropic media into the elliptic anisotropic operator (free of the artifacts) and the non-elliptic-anisotropic components, which form a pseudo-differential operator. We, then, develop a separable approximation of the dispersion relation of non-elliptic-anisotropic components, specifically for transversely isotropic (TI) media. Finally, we iteratively solve the simpler lower-order elliptical wave equation for a modified source function that includes the non-elliptical terms represented in the Fourier domain. A frequency domain Helmholtz
\end{abstract}


${ }_{21}$ formulation of the approach renders the iterative implementation efficient as the cost is 22 dominated by the Lower-Upper (LU) decomposition of the impedance matrix for the sim23 pler elliptical anisotropic model. Also, the resulting wavefield is free of S-wave artifacts and 24 has balanced amplitude. Numerical examples show that the method is reasonably accurate 25 and efficient. 


\section{INTRODUCTION}

The acoustic approximation is widely used in current industry imaging and inversion algorithms(Gholami et al., 2013; Alkhalifah and Plessix, 2014; Cheng et al., 2014a,b; Operto et al., 2015; da Silva et al., 2016). This approximation was introduced by Alkhalifah (2000) for the purpose of resolving quasi-P wave propagation in transversely isotropic (TI) media. In his approach, the shear-wave velocity along the symmetry axis is set to zero, resulting in a scalar fourth-order differential equation. Zhou et al. (2006) decomposed the fourthorder differential equation into a coupled system of second-order differential equations, and proposed a computationally efficient scheme. After that, several variations (Bakker and Duveneck, 2011; Zhang et al., 2011; Bube et al., 2012) were proposed. However, most of them are based on modeling in the time domain. We can also use Fourier transformation to obtain the related equations in the frequency domain from the time domain; however, the resulting algorithm usually contains $S$-wave artifacts. Another family of high resolution algorithms for solving the anisotropic acoustic wave equation without $S$-wave artifacts falls under the so-called spectral approach (Etgen and Brandsberg-Dahl, 2009; Du et al., 2010; Fomel et al., 2013; Alkhalifah, 2014; Song and Alkhalifah, 2013; Wu and Alkhalifah, 2014; Sun et al., 2016). However, they are relatively expensive and difficult to extend to the frequency domain.

There has been much less work done on forward modeling in the frequency domain in anisotropic media, compared to the time domain. Operto et al. (2009) recast the wave equation as a system of two second-order wave equations for the pressure wavefield and an auxiliary wavefield accounting for anellipticity. Chu and Stoffa (2012) proposed new compact finite difference operators for pseudo-acoustic and pure acoustic wave equations for 
vertical transversely isotropic (VTI) media in the frequency domain. Wang et al. (2012a,b) proposed a massively parallel structured direct solver to improve the efficiency of LU decomposition. Operto et al. (2014) presented a 3D visco-acoustic finite difference frequency domain method performing seismic modeling in VTI media. However, most existing methods in the frequency domain suffer from S-wave artifacts. These artifacts are reduced when the source is located in the isotropic region (Alkhalifah, 2000), but become unacceptable when the source is located in the anisotropic region or we have strong scattering acting as secondary sources.

Another group of efficient algorithms for computing pure quasi-P waves is called effective isotropic model approximations (Alkhalifah et al., 2013; Ibanez-Jacome et al., 2014; Waheed and Alkhalifah, 2015). These approaches perform the quasi-P wave calculation in two steps: first by solving the Eikonal equation from an anisotropic quasi-P wave velocity model, thereby obtaining the propagation direction at each spatial point; this allows the determination of the phase velocity and the formation of an effective model for quasi-P wave propagation. Then the isotropic wave equation is solved using finite differences with the effective model. The computational cost of the effective isotropic model approach is close to that of solving an isotropic acoustic wave equation.

Recently, Xu and Zhou (2014) proposed a new acoustic-like equation that decompose the original pseudo-differential operator into two numerically solvable operators: a Laplacian operator and a scalar operator. The combination of these two operators yields an accurate phase for quasi-P wave propagation. This solution is shear-wave free and numerically stable even for complicated anisotropic models. Since only one equation is required to obtain a numerical solution, the new proposed scheme is more efficient than conventional schemes that solve a system of second-order differential equations. In order to compensate for 
amplitude errors, Xu et al. (2015) proposed decomposing the original operator into elliptic anisotropic and anelliptic anisotropic components. Zhang et al. (2017) proposed a method to compensate for the amplitude based on an isotropic background. In order to reduce the cost, they suggest doing so in the time domain. Le et al. (2015) applied their method to full waveform inversion.

In this paper, we aim to derive an efficient implementation of wave propagation in VTI media in the frequency domain without S-wave artifact. We first propose a general framework to derive an anisotropic formulations of the wave equation, which allows us to iteratively solve it using simplified anisotropic or isotropic linear wave equations based on the fixed-point method or any other advanced iterative method (Saad, 2003). After that, we separate the dispersion relation for acoustic media into elliptic-anisotropic components and non-elliptic-anisotropic components. Then, we obtain a separable approximation for the non-elliptic-anisotropic components. At last, we apply the above mentioned iterative framework to the approximated dispersion and obtain an efficient method for solving the Helmholtz equation in acoustic VTI media.

\section{PSEUDO-DIFFERENTIAL EQUATION AND ITS SOLUTION}

Acoustic wave propagation in general anisotropic media can be described as a pseudodifferential equation (Alkhalifah, 2000). Let us formulate the dispersion relation for general anisotropic media as

$$
L(\vec{k}, \vec{p}, \omega)=0,
$$

in which, $\vec{k}=\left\{k_{x}, k_{y}, k_{z}\right\}$ is the wave number, $\vec{p}$ represents the material parameters (at this stage stationary with space) and $\omega$ is the angular frequency. Different operators $L$ 
will describe different anisotropic assumptions of the Earth. Since the operator $L$ is not always a polynomial function, the corresponding partial-differential equation (PDE) might be nonlinear. In most situations, the operator $L$ can be divided into two parts:

$$
L(\vec{k}, \vec{p}, \omega)=L_{1}\left(\vec{k}, \vec{p}_{0}, \omega\right)+L_{2}\left(\vec{k}, \vec{p}, \vec{p}_{0}, \omega\right)
$$

where we assume that $L_{1}$ is a reasonably good approximation of $L$, that the inversion of $L_{1}$ can be easily obtained and that $\vec{p}-\vec{p}_{0}$ is relatively small. In our case, we assume that $L_{1}$ is a polynomial, which means that $L_{1}$ is a linear partial differential equation. We transform the above dispersion relation into a partial differential equation (using inverse Fourier transform from wavenumbers to space coordinates):

$$
L_{1}\left(u, \vec{p}_{0}, \omega\right)+L_{2}\left(u, \vec{p}, \vec{p}_{0}, \omega\right)=f
$$

where $f$ is a given source function. This provides us with an easy way for solving the above nonlinear equation using the fixed-point iterative method. Provided $u^{i}$, the solution of the iteration $i+1$ can be calculated by solving the following equation:

$$
L_{1}\left(u^{i+1}, \vec{p}_{0}, \omega\right)=f-L_{2}\left(u^{i}, \vec{p}, \vec{p}_{0}, \omega\right) .
$$

Solving the equation above is usually much simpler and cheaper than solving the original anisotropic equation. Since the cost of LU decomposition to obtain $L_{1}^{-1}$ is much higher than applying the matrix-vector multiply $L_{1}^{-1} f$, the resulting algorithm has almost the same cost as the LU decomposition to obtain $L_{1}^{-1}$, even though we need to complete several iterations to obtain the solution. Of course, other methods other than fixed-point scheme for solving 
the nonlinear equation can be utilized to speed up the convergence. The initial guess $u^{0}$ can be easily obtained by setting $L_{2}=0$ and solving the following equation:

$$
L_{1}\left(u^{0}, \vec{p}_{0}, \omega\right)=f
$$

We summarize the above method for solving the general acoustic anisotropic wave equation in Algorithm 1.

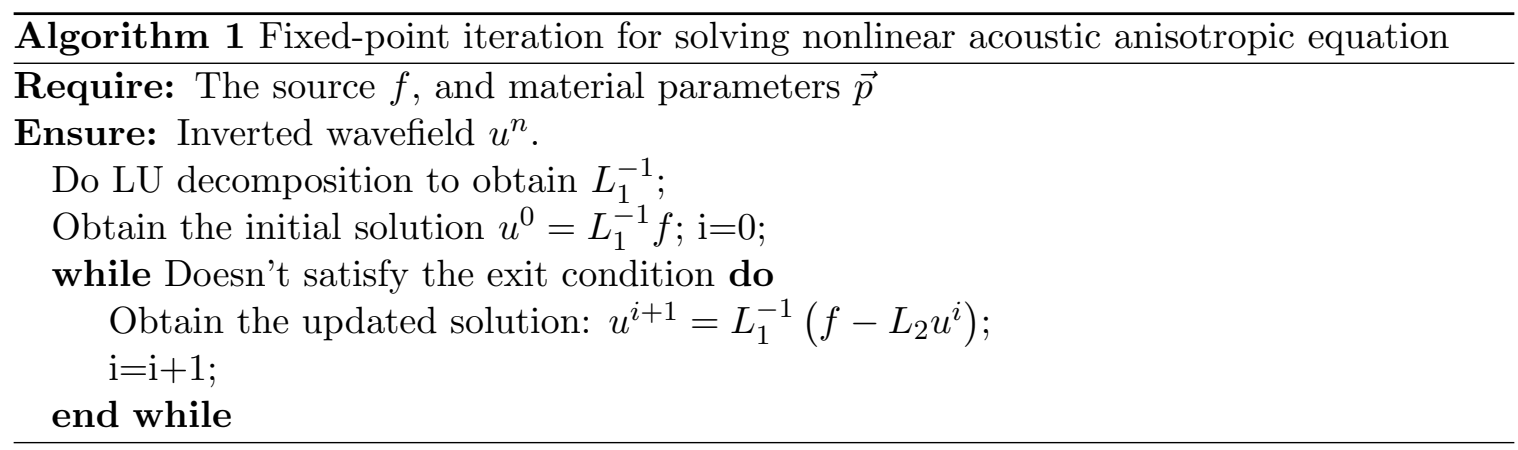
(1)

More generally, we can consider the above mentioned method as a preconditioned system under a general iterative framework:

$$
L_{1}^{-1}\left(u, \vec{p}_{0}, \omega\right)\left(L_{1}\left(u, \vec{p}_{0}, \omega\right)+L_{2}\left(u, \vec{p}, \vec{p}_{0}, \omega\right)\right)=L_{1}^{-1}\left(u, \vec{p}_{0}, \omega\right) f
$$

For most of the implementation of the existing iterative method, we only need a matrix free implementation of the operator(Saad, 2003). In stead of using $L_{1}\left(u, \vec{p}_{0}, \omega\right)+$ $L_{2}\left(u, \vec{p}, \vec{p}_{0}, \omega\right)$, which will take a lot of iteration, we suggest to insert the preconditioned system $L_{1}^{-1}\left(u, \vec{p}_{0}, \omega\right)\left(L_{1}\left(u, \vec{p}_{0}, \omega\right)+L_{2}\left(u, \vec{p}, \vec{p}_{0}, \omega\right)\right)$ into the general iterative method. One advantage of the above mentioned method is that the discretization scheme of $L_{1}$ and $L_{2}$ can be totally different. For $L_{1}$, we can approximate it by finite difference method. For $L_{2}$, we can use any discretization scheme such as high order finite difference method or even 
spectral method, because we do not need to know the inverse operator of $L_{2}$ in the above mentioned algorithm. In this case, we can combine two different discrete scheme in one algorithm to solve the continuous equation. This is synonymous with spectral methods in the time domain mentioned above.

\section{A SEPARABLE APPROXIMATION TO VTI MEDIA}

In the previous section, we proposed a new framework for solving a general anisotropic wave equation. Under this framework, we can easily combine different discretization methods for different components of the equation. However, to implement the above mentioned method, we need an efficient implementation of $L_{2}\left(u, \vec{p}, \vec{p}_{0}, \omega\right)$. In order to do that, we demonstrate how to obtain a separable approximation of the original dispersion relation for acoustic VTI media in this section. After this separable approximation, we can use spectral methods for the discretization of the non-elliptic-anisotropic components.

The dispersion relation for acoustic VTI media in the frequency-wavenumber domain (Alkhalifah, 2000) can be represented as follows:

$$
\omega^{2}=\frac{v^{2}}{2}\left((1+2 \epsilon) k_{x}^{2}+k_{z}^{2}+\sqrt{\left((1+2 \epsilon) k_{x}^{2}+k_{z}^{2}\right)^{2}-8(\epsilon-\delta) k_{x}^{2} k_{z}^{2}}\right)
$$

in which, $\omega$ is the angular frequency, $\epsilon$ and $\delta$ are the familiar Thomsen parameters and $v$ is the velocity along symmetry axis. The spatial wavenumber vector $\vec{k}$ is, as usual, defined as $\vec{k}=\left\{k_{x}, k_{z}\right\}$ in $2 \mathrm{D}$ media. We can reformulate the dispersion relation as:

$$
\omega^{2}=(1+F)\left(v^{2} k_{z}^{2}+v^{2}(1+2 \epsilon) k_{x}^{2}\right)
$$


with $F$ given by

$$
F(\vec{k}, \vec{p})=\frac{1}{2}\left(\sqrt{1-\frac{8(\epsilon-\delta) k_{x}^{2} k_{z}^{2}}{\left((1+2 \epsilon) k_{x}^{2}+k_{z}^{2}\right)^{2}}}-1\right) .
$$

Let us denote

$$
m=\frac{8(\epsilon-\delta) k_{x}^{2} k_{z}^{2}}{\left(k_{x}^{2}+k_{z}^{2}\right)^{2}}, n=\frac{2 \epsilon k_{x}^{2}}{\left(k_{x}^{2}+k_{z}^{2}\right)} .
$$

Then

$$
F=\frac{1}{2}\left(\sqrt{1-\frac{m}{(1+n)^{2}}}-1\right)
$$

The key problem in the above formulation is that $F$ is a function of both space and wavenumber. In this case, we can not simply utilize fast Fourier transform for operator $F$. Thus, we need to approximate $\mathrm{F}$ with some kind of separable form. To do that, we seek the following approximation of $F$ :

$$
F_{a}=b_{1} m^{b_{2}}+b_{3} m^{b_{4}} n^{b_{5}} .
$$

In the above formulation, $b_{1}, b_{2}, b_{3}, b_{4}, b_{5}$ are the coefficients to be decided. Actually, we can choose $b_{1}=-\frac{1}{4}, b_{2}=1, b_{3}=-0.5, b_{4}=1, b_{5}=1$, then

$$
F_{a}=-\frac{m}{4}+\frac{m n}{2}
$$

which reduces to a Taylor series expansion of $F$ over the two variable $m$ and $n$. It is a good approximation of $F$ when $m$ and $n$ are relatively small. To make the approximation more accurate for a larger range of $m$ and $n$, we need to search for the best $\left\{b_{i}\right\}_{i=1}^{5}$. Considering there is some constrain $(m, n) \in \Omega$, then the coefficients can be decided through solving the 
following optimization problem:

$$
\min _{b_{1}, b_{2}, b_{3}, b_{4}, b_{5}} \max _{(m, n) \in \Omega}\left|F-F_{a}\right| .
$$

Here we assume that $b_{2}>0$ and $b_{4}>0$. Then $F_{a}=0$ when $m=0$. In this special case, the original dispersion relation represents the elliptic anisotropic wave equation and our approximation dispersion relation is the same as the original dispersion $F\left(F_{a}=F\right)$. It indicates that there is no approximation in the case of elliptic anisotropic wave equation. The accuracy of the approximation is demonstrated in Table 1 for different ranges of $m$ and $n$. Compared with the standard Taylor series expansion, the optimal coefficients will be much more accurate for the same range of $m$ and $n$, especially for relatively large $m$ and $n$. Comparing the dispersion error of the optimal finite difference method (Jo et al., 1996) even for isotropic wave equation, which is about 0.005 , our maximum dispersion error is negligible for even $m \leq 0.5$ and $n \leq 0.5$. To compare more, we show the exact $F$ for different $m$ and $n$ in Figure 1(a). The error of the Taylor based (proposed) approximation is shown in Figure 1(b) (Figure 1(c)). We can see from Figure 1(b) and 1(c) that the proposed approximation is much more accurate than the Taylor series expansion based approximation. Figures 1(a), 1(b), and 1(c) are plotted using the same scale. To show the error distribution, we multiply the error distribution of the proposed method by 10 and show it in Figure 1(d).

At last, the choice of $\Omega$ can be decided by the range of $\epsilon$ and $\delta$. If $\epsilon-\delta \leq \alpha$ for a given $\alpha$, then $m=\frac{8(\epsilon-\delta) k_{x}^{2} k_{z}^{2}}{\left(k_{x}^{2}+k_{z}^{2}\right)^{2}} \leq 2 \alpha$. If $\epsilon \leq \beta$, then $n=\frac{2 \epsilon k_{x}^{2}}{\left(k_{x}^{2}+k_{z}^{2}\right)} \leq 2 \beta$. 


\section{THE NUMERICAL ALGORITHM FOR SOLVING THE ACOUSTIC WAVE EQUATION IN VTI MEDIA}

Since we have obtained a separable approximation, we will format the approximate dispersion relation into a framework that allows us to obtain a numerical algorithm for solving the wave equation in acoustic VTI media. According to the above derivation, we can set:

$$
\begin{aligned}
& L_{1}(\vec{k}, \vec{p}, \omega)=\omega^{2}-\left(v^{2} k_{z}^{2}+v^{2}(1+2 \epsilon) k_{x}^{2}\right), \\
& L_{2}(\vec{k}, \vec{p}, \omega)=-F_{a}(\vec{k}, \vec{p})\left(v^{2} k_{z}^{2}+v^{2}(1+2 \epsilon) k_{x}^{2}\right) .
\end{aligned}
$$

The component $L_{1}$ can be easily approximated using finite difference approximation. However, the components $F_{a}$ can not be approximated with high accuracy using finite difference. Since it is a separable approximation of $F$, it can be easily implemented using instead fast Fourier transforms. Let us first assume that

$$
m(\vec{x}, \vec{k})=m_{x}(\vec{x}) m_{k}(\vec{k}), n(\vec{x}, \vec{k})=n_{x}(\vec{x}) n_{k}(\vec{k})
$$

According to the definition in (12),

$$
F_{a}=b_{1} m^{b_{2}}+b_{3} m^{b_{4}} n^{b_{5}}=b_{1} m_{x}(\vec{x})^{b_{2}} m_{k}(\vec{k})^{b_{2}}+b_{3} m_{x}(\vec{x})^{b_{4}} n_{x}(\vec{x})^{b_{5}} m_{k}(\vec{k})^{b_{4}} n_{k}(\vec{k})^{b_{5}} .
$$

Thus, to implement $L_{2}(\vec{k}, \vec{p}, \omega) u$ for a given discrete wavefield $u$, we first obtain the approximation function $f_{h} \approx\left(v^{2} \frac{\partial^{2} u}{\partial z^{2}}+v^{2}(1+2 \epsilon) \frac{\partial^{2} u}{\partial x^{2}}\right)$. The approximation function $f_{h}$ can be easily obtained using the finite difference method. Let $\mathbf{F}$ be the discrete Fourier transform operator and $\mathbf{F}^{-1}$ be the inverse Fourier transform. Then $L_{2}(\vec{k}, \vec{p}, \omega) u$ can be represented 
as:

$$
b_{1} m_{x}(\vec{x})^{b_{2}} \mathbf{F}^{-1}\left(m_{k}(\vec{k})^{b_{2}} \mathbf{F}\left(f_{h}\right)\right)+b_{3} m_{x}(\vec{x})^{b_{4}} n_{x}(\vec{x})^{b_{5}} \mathbf{F}^{-1}\left(m_{k}(\vec{k})^{b_{4}} n_{k}(\vec{k})^{b_{5}} \mathbf{F}\left(f_{h}\right)\right) .
$$

In this case, we need one forward and two inverse Fourier transforms for calculating $L_{2}(\vec{k}, \vec{p}, \omega) u$. Pay attention to that the cost of a fast Fourier transform is $O(N \log (N))$, which is far less than the cost to obtain $L_{1}^{-1}$, which is $O\left(N^{2}\right)$ for the sparse matrix obtained by finite difference method.

\section{NUMERICAL EXAMPLES}

In this section, for a possible application in inversion, we consider the parameter $\vec{p}=\left\{v_{h}=v(1+2 \epsilon), \eta=\frac{\epsilon-\delta}{1+2 \delta}, \epsilon\right\}$ to reduce the crosstalk between the different parameters, according to the radiation pattern analysis (Alkhalifah and Plessix, 2014). The first example shows the accuracy of the proposed method when solving the nonlinear partial differential equation. Figures 2(a) and 2(b) show the real and imaginary parts of the wavefield, respectively, with frequency $f=10 \mathrm{~Hz}, v_{h}=1.8 \mathrm{~km} / \mathrm{s}, \epsilon=0.2$ and $\eta=0$, which corresponds to an elliptic anisotropic medium. Figures 2(c) and 2(d) show the real and imaginary parts of the wavefield with $v_{h}=1.8 \mathrm{~km} / \mathrm{s}, \epsilon=0.2$ and $\eta=0.2$ after 20 iterations. To demonstrate the accuracy of the wavefield, we compute the wavefield for each frequency and obtain the corresponding time domain wavefield. The snapshot at $1.6 \mathrm{~s}$ using the proposed method is shown in Figure 3(a) for $\eta=0$ and 3(b) for $\eta=0.2$. To compare, we display the snapshot at 1.6s using the low-rank spectral (Fomel et al., 2013) approach, shown in Figure 3(c) for $\eta=0$ and $3(\mathrm{~d})$ for $\eta=0.2$. Meanwhile, the snapshots at $1.6 \mathrm{~s}$ using the standard finitedifference implementation of acoustic equation (Bakker and Duveneck, 2011) are shown in 
Figures 3(e) for $\eta=0$, and 3(f) for $\eta=0.2$. As we can see, the proposed method produces a reasonably accurate solution. Also, there are no S-wave artifacts for both the low-rank method and the proposed method while the standard finite difference implementation suffers from S-wave artifacts. To make the comparison clearer, we show the wavefield profile at $x=2 \mathrm{~km}$ in Figure 4 . It indicates that the proposed method is reasonably accurate.

Before investigating more complicated models, we apply the proposed method to a model with an interface. The velocity of the upper layer is $1.5 \mathrm{~km} / \mathrm{s}$ and the velocity of the lower layer is $1.8 \mathrm{~km} / \mathrm{s}$. The $\eta$ in the upper layer is 0.25 and the $\eta$ lower layer is 0.2 . Figures 5(a) and5(b) show the real and imaginary components of the wavefield with $\eta=0$. Figures $5(\mathrm{c})$ and $5(\mathrm{~d})$ show the real and imaginary components of the wavefield with the actual $\eta$. According to these figures, our approach managed to perturb the wavefield. To evaluate the accuracy of the method, we sum the wavefields for all the frequencies and obtain a snapshot of the wavefield in time. Figure 6(a) shows the modeled wavefield with $\eta=0$ using the new method. Figure 6(b) shows the modeled wavefield with $\eta=0$ using the low-rank time domain approach. Figure 6(c) shows the modeled wavefield with the actual $\eta$ using the new method. Figure 6(d) shows the modeled wavefield with the actual $\eta$ using the low-rank time domain approach. Our method produced results that are similar to those of the time domain low-rank method, and free of shear wave artifacts.

Next, we compare the accuracy for more complicated models. We specifically utilize part of the BP2007 anisotropic model. The horizontal velocity $v_{x}$, anisotropic parameters $\eta$ and $\epsilon$ are shown in Figures $7(\mathrm{a}), 7(\mathrm{~b})$ and $7(\mathrm{c})$, respectively. We place a source at 
$\left(x_{s}, z_{s}\right)=(4.5 \mathrm{~km}, 8 \mathrm{~km})$ and use a space sampling of $0.0125 \mathrm{~km}$. We sum the wavefield of all the frequencies and obtain the wavefield in the time space domain. A snapshot of the wavefield of the proposed method and low-rank method are shown in Figures $8(\mathrm{a})$ and 8(b) for comparison. To show the accuracy, we overlay the first-arrival travel time solution. We can see that the proposed method can also deal with a complicated model.

To show the method can be easily extended to three dimensions, we consider a simple constant-parameters model $\left(v_{h}=1.8 \mathrm{~km} / \mathrm{s}, \eta=0.2\right.$, and $\left.\epsilon=0.2\right)$. The source is located in the middle of the model. We sum all the wavefield of all the frequencies used and obtain the wavefield in time-space domain. The snapshot at $t=0.5 \mathrm{~s}$ is shown in Figure 9 .

At last, we apply reverse time migration on a portion of the HESS VTI model. The material parameters $\left(v_{x}, \eta, \epsilon\right)$ are shown in Figures $12(\mathrm{a}), 12(\mathrm{~b})$ and $12(\mathrm{c})$, respectively. Using the low-rank method, we generate 93 shot gathers with a shot sampling of $0.1 \mathrm{~km}$ located on the surface. The maximum offset for each shot is $3.75 \mathrm{~km}$. Figure 10(a) shows the data modeled using the low-rank method for the central shot. For comparison, we plot the data modeled using the proposed method for the same shot in Figure 10(b). The resulting shot gathers are similar. To demonstrate the accuracy, we overlay the traces at the offset of $1.0 \mathrm{~km}$ obtained by low-rank method and the proposed method in Figure 11. Using the data generated with the low-rank modeling approach, we use the proposed method as the engine of reverse time migration. The resulting RTM image is shown in Figure 12(d). The accuracy of the proposed method is reflected in the clean reverse time migration image. 


\section{CONCLUSIONS}

We have proposed an efficient solution for the acoustic Helmholtz wave equation in VTI media. We first separate the pseudo-differential operator for acoustic VTI media into ellipticanisotropic component and the non-elliptic-anisotropic component. After that, we derived a reasonably accurate separable approximation of the non-elliptic-anisotropic component, which makes it possible to implement it using Fast Fourier transform methods. At last, we combine the finite difference approximation of the elliptic anisotropic component and spectral approximation of the non-elliptic-anisotropic component in an iterative framework. The solution of the resulting Helmholtz formulation is free of $S$-wave artifacts and has well balanced amplitude. The resulting algorithm has almost identical cost to that of solving the Helmholtz equation in elliptic-anisotropic media.

\section{ACKNOWLEDGMENTS}

We thank KAUST for its support and the SWAG group for the collaborative environment. Especially, we thank Zhendong Zhang for useful discussions. We also thank Hemang Shah, Faqi Liu, Scott Morton, Hess Corporation and BP Exploration Operation for providing the benchmark model. The research reported in this publication was supported by funding from King Abdullah University of Science and Technology (KAUST). For computer time, this research used the resources of the Supercomputing Laboratory at King Abdullah University of Science and Technology (KAUST) in Thuwal, Saudi Arabia. We also thank the associate editor Dimitri Komatitsch, assistant editor Arthur Cheng, Jiubing Cheng and another 
258 anonymous reviewer for their fruitful suggestions and comments. 


\section{REFERENCES}

Alkhalifah, T., 2000, An acoustic wave equation for anisotropic media: GEOPHYSICS, 65, $1239-1250$.

—, 2014 , Effective wavefield extrapolation in anisotropic media: accounting for resolvable anisotropy: Geophysical Prospecting, 62, 1089-1099.

Alkhalifah, T., X. Ma, U. bin Waheed, and M. Zuberi, 2013, Efficient anisotropic wavefield extrapolation using effective isotropic models: 75th Annual EAGE Meeting, EAGE, Expanded Abstracts, Tu0116.

Alkhalifah, T., and R. Plessix, 2014, A recipe for practical full-waveform inversion in anisotropic media: An analytical parameter resolution study: GEOPHYSICS, 79, no. 3, R91-R101.

Bakker, P. M., and E. Duveneck, 2011, Stability analysis for acoustic wave propagation in tilted ti media by finite differences: Geophysical Journal International, 185, 911-921.

Bube, K. P., T. Nemeth, J. P. Stefani, R. Ergas, W. Liu, K. T. Nihei, and L. Zhang, 2012, On the instability in second-order systems for acoustic VTI and TTI media: GEOPHYSICS, 77, no. 5, T171-T186.

Cheng, X., K. Jiao, D. Sun, and D. Vigh, 2014a, Anisotropic parameter estimation with full-waveform inversion of surface seismic data: 84th Annual International Meeting, SEG, Expanded Abstracts, 1072-1077.

— , 2014b, Multiparameter full-waveform inversion for acoustic VTI medium with surface seismic data: 76th Annual EAGE Meeting, EAGE, Expanded Abstracts, WeE10604.

Chu, C., and P. L. Stoffa, 2012, Pure and pseudo-acoustic VTI wave modeling in the frequency domain using compact finite difference operators: 82th Annual International Meeting, SEG, Expanded Abstracts, 1-5. 
da Silva, N. V., A. Ratcliffe, V. Vinje, and G. Conroy, 2016, A new parameter set for anisotropic multiparameter full-waveform inversion and application to a north sea data set: GEOPHYSICS, 81, no. 4, U25-U38.

Du, X., R. P. Fletcher, and P. J. Fowler, 2010, Pure P-wave propagators versus pseudoacoustic propagators for RTM in VTI meida: 72nd Annual EAGE Meeting, EAGE, Expanded Abstracts, C013.

Etgen, J., and S. Brandsberg-Dahl, 2009, The pseudo-analytical method: application of pseudo-Laplacians to acoustic and acoustic anisotropic wave propagation: 79th Annual International Meeting, SEG, Expanded Abstracts, 2552-2556.

Fomel, S., L. Ying, and X. Song, 2013, Seismic wave extrapolation using lowrank symbol approximation: Geophysical Prospecting, 61, 526-536.

Gholami, Y., R. Brossier, S. Operto, A. Ribodetti, and J. Virieux, 2013, Which parameterization is suitable for acoustic vertical transverse isotropic full waveform inversion? part 1: Sensitivity and trade-off analysis: GEOPHYSICS, 78, no. 2, R81-R105.

Ibanez-Jacome, W., T. Alkhalifah, and U. b. Waheed, 2014, Effective orthorhombic anisotropic models for wavefield extrapolation: Geophysical Journal International, 198, $1653-1661$.

Jo, C., C. Shin, and J. H. Suh, 1996, An optimal 9point, finitedifference, frequencyspace, 2-d scalar wave extrapolator: GEOPHYSICS, 61, 529-537.

Le, H., B. Biondi, R. G. Clapp, and S. A. Levin, 2015, Using a nonlinear acoustic wave equation for anisotropic inversion: 85th Annual International Meeting, SEG, Expanded Abstracts, 467-471.

Operto, S., R. Brossier, L. Combe, L. Mtivier, A. Ribodetti, and J. Virieux, 2014, Computationally efficient three-dimensional acoustic finite-difference frequency-domain seis- 
mic modeling in vertical transversely isotropic media with sparse direct solver: GEOPHYSICS, 79, no. 5, T257-T275.

Operto, S., A. Miniussi, R. Brossier, L. Combe, L. Mtivier, V. Monteiller, A. Ribodetti, and J. Virieux, 2015, Efficient 3-d frequency-domain mono-parameter full-waveform inversion of ocean-bottom cable data: application to valhall in the visco-acoustic vertical transverse isotropic approximation: Geophysical Journal International, 202, 1362-1391.

Operto, S., J. Virieux, A. Ribodetti, and J. E. Anderson, 2009, Finite-difference frequencydomain modeling of viscoacoustic wave propagation in $2 \mathrm{D}$ tilted transversely isotropic (tti) media: GEOPHYSICS, 74, no. 5, T75-T95.

Saad, Y., 2003, Iterative methods for sparse linear systems: SIAM.

Song, X., and T. Alkhalifah, 2013, Modeling of pseudoacoustic p-waves in orthorhombic media with a low-rank approximation: GEOPHYSICS, 78, no. 4, C33-C40.

Sun, J., S. Fomel, and L. Ying, 2016, Low-rank one-step wave extrapolation for reverse time migration: GEOPHYSICS, 81, no. 1, S39-S54.

Waheed, U. b., and T. Alkhalifah, 2015, An efficient wave extrapolation method for anisotropic media with tilt: Geophysical Prospecting, 63, 1126-1141.

Wang, S., M. V. de Hoop, J. Xia, and X. S. Li, 2012a, Massively parallel structured multifrontal solver for time-harmonic elastic waves in 3-D anisotropic media: Geophysical Journal International, 191, 346-366.

Wang, S., J. Xia, M. V. de Hoop, and X. S. Li, 2012b, Massively parallel structured direct solver for equations describing time-harmonic qp-polarized waves in TTI media: Geophysics, 77, no. 3, T69-T82.

Wu, Z., and T. Alkhalifah, 2014, The optimized expansion based low-rank method for wavefield extrapolation: GEOPHYSICS, 79, no. 2, T51-T60. 
Xu, S., B. Tang, J. Mu, and H. Zhou, 2015, Quasi-p wave propagation with an elliptic differential operator: 85th Annual International Meeting, SEG, Expanded Abstracts, $4380-4384$.

$\mathrm{Xu}$, S., and H. Zhou, 2014, Accurate simulations of pure quasi-p-waves in complex anisotropic media: GEOPHYSICS, 79, no. 6, T341-T348.

Zhang, Y., H. Zhang, and G. Zhang, 2011, A stable TTI reverse time migration and its implementation: GEOPHYSICS, 76, no. 3, WA3-WA11.

Zhang, Z.-D., Y. Liu, T. Alkhalifah, and Z. Wu, 2017, Efficient anisotropic quasi-p wavefield extrapolation using an isotropic low-rank approximation: Geophysical Journal International, submitted.

Zhou, H., G. Zhang, and R. Bloor, 2006, An anisotropic acoustic wave equation for VTI media: 68th Annual EAGE Meeting, EAGE, Expanded Abstracts, H033. 